\title{
Intracoronary near-infrared spectroscopy-role and clinical applications
}

\author{
Stephen E. Wilkinson, Ryan D. Madder \\ Frederik Meijer Heart \& Vascular Institute, Spectrum Health, Grand Rapids, MI, USA \\ Contributions: (I) Conception and design: RD Madder; (II) Administrative support: SE Wilkinson; (III) Provision of study materials or patients: RD \\ Madder; (IV) Collection and assembly of data: All authors; (V) Data analysis and interpretation: All authors; (VI) Manuscript writing: All authors; (VII) \\ Final approval of manuscript: All authors. \\ Correspondence to: Ryan D. Madder, MD. Frederik Meijer Heart \& Vascular Institute, Spectrum Health, 100 Michigan Street NE, Grand Rapids, MI, \\ USA. Email: ryan.madder@spectrumhealth.org.
}

\begin{abstract}
Intracoronary near-infrared spectroscopy (NIRS) has been developed and validated for the detection of lipid-rich plaque in the coronary arteries. A combined NIRS and intravascular ultrasound catheter is currently in clinical use and has an emerging role in evaluating plaques both before and after percutaneous coronary intervention. NIRS has recently been shown to positively identify both vulnerable patients and vulnerable plaques. This review focuses on the principles and image interpretation of intracoronary NIRS, as well as its clinical applications, limitations, and future directions.
\end{abstract}

Keywords: Intracoronary imaging; lipid-rich plaque (LRP); near-infrared spectroscopy (NIRS); percutaneous coronary intervention (PCI)

Submitted Dec 14, 2019. Accepted for publication Feb 03, 2020.

doi: $10.21037 / \mathrm{cdt} .2020 .02 .02$

View this article at: http://dx.doi.org/10.21037/cdt.2020.02.02

\section{Introduction}

Intracoronary near-infrared spectroscopy (NIRS) has been developed and validated for the detection of lipidrich plaque (LRP), and is currently in clinical use with a multimodality catheter that combines NIRS with intravascular ultrasound (IVUS). This review describes the principles of NIRS, intracoronary NIRS image interpretation, and the clinical applications of NIRS both before and after percutaneous coronary intervention (PCI). An overview of the ability of NIRS to identify vulnerable patients and vulnerable plaques is also presented, and the limitations of NIRS and possible future directions are discussed.

\section{Principles of NIRS}

NIRS utilizes electromagnetic radiation with frequencies slightly lower than the visible spectrum to characterize the chemical composition of materials, including tissues. To do this, a light source emits near-infrared light towards a target. The proportion of diffusely reflected light returning to a detector is then analyzed as a function of wavelength (1). Based on the specific spectral signals produced in this process, the chemical composition of the target can be determined. Because different tissues have unique chemical compositions, NIRS can differentiate them based upon their specific spectral signatures.

Owing to its ability to differentiate tissue types, NIRS has many clinical applications in medicine, including an emerging role in the evaluation of coronary arteries. More specifically, intracoronary NIRS has been developed and extensively validated against the gold standard of histology to identify the presence of LRP within coronary arteries (2). The ability to identify LRP based on its spectral signature differentiates NIRS from other intracoronary imaging modalities, such as IVUS and optical coherence tomography (OCT), which largely provide plaque structural characterizations, and can only infer the presence of LRP based on signal attenuation. Signal attenuation in IVUS and OCT 


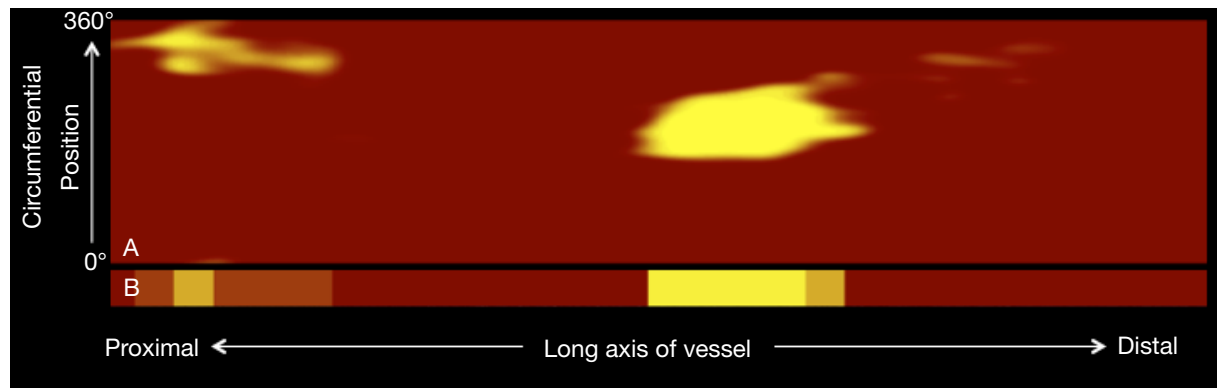

Figure 1 Interpretation of the NIRS chemogram and block chemogram. An example of a NIRS chemogram (A) and block chemogram (B) are shown. The $\mathrm{X}$-axis of the chemogram represents the spatial location along the long axis of the vessel. The Y-axis of the chemogram represents circumferential position. Red regions of the chemogram correspond to locations having a low probability of LRP. Yellow regions of the chemogram correspond to locations having a higher probability of LRP. In this example NIRS has detected a moderate size LRP in the mid-segment and a small LRP in the proximal segment of the vessel. LRP, lipid-rich plaque; NIRS, near-infrared spectroscopy.

imaging is not specific to LRP alone, as a variety of factors can attenuate their signal, thereby reducing the predictive value of LRP detection by these imaging modalities $(3,4)$.

\section{NIRS catheter and image interpretation}

Intracoronary NIRS is performed with an imaging catheter delivered to the distal portion of a target vessel over a standard coronary guidewire. At the tip of the catheter, near-infrared light is emitted in a diffuse, non-focused manner. During the imaging process, the light source is rapidly rotated while the catheter is automatically withdrawn through the vessel by motorized pullback, thereby capturing images over the full circumference and length of the imaged vessel. The variable wavelengths of emitted light are either absorbed into the tissues or scattered. Returning scattered spectral signals are detected by the catheter and transmitted to a computer housed in a bedside console. In this manner, approximately 30,000 measurements of spectral signals are acquired per $100 \mathrm{~mm}$ of scanned artery (5).

Software within the console analyzes the captured spectral data to determine the probability of LRP at each imaged location within the vessel. Based on these probabilities, the software produces a color-coded graphical representation of the chemical composition of interrogated tissue called a chemogram. The chemogram is essentially a map of the imaged vessel, with the $\mathrm{X}$-axis representing axial position along the length of the artery, and the Y-axis representing circumferential position. The chemogram is comprised of pixels, with each pixel being assigned a color on a 128 -color scale spanning from red to yellow based on the probability of LRP at that location. Pixels with a probability of LRP exceeding 0.6 appear yellow, whereas pixels corresponding to anatomic locations with a probability of LRP $<0.6$ appear red (Figure 1A) (6). In addition to the chemogram, the NIRS software produces a block chemogram comprised of $2 \mathrm{~mm}$ blocks spanning the axial length of the imaged vessel. Each 2-mm block corresponds to a $2 \mathrm{~mm}$ section of artery and is assigned one of four colors based on the $90^{\text {th }}$ percentile probability value of LRP of all pixels in the block. In this manner, the block chemogram provides an easy to read visualization of the probability of LRP within corresponding sections of artery by a color code of red (probability $<0.57$ ), orange (probability 0.57 to $<0.84$ ), $\tan$ (probability 0.84 to $\leq 0.98$ ), and yellow (probability >0.98) (Figure 1B) (6).

In addition to providing the probability of LRP at each location within an imaged artery, intracoronary NIRS also provides a semi-quantitative estimate of the amount of lipid using a metric termed the lipid core burden index (LCBI) (Figure 2). The LCBI in any selected region of interest is defined as the number of pixels with a probability of lipid $>0.6$ divided by the total number of valid pixels, with the quotient multiplied by 1,000 (2). Thus, LCBI values range from 0 to 1,000 . The NIRS software can also automatically identify the maximum LCBI in any 4-mm segment within a larger region of interest. This metric is termed the maximum LCBI in $4 \mathrm{~mm}\left(\operatorname{maxLCBI}{ }_{4 \mathrm{~mm}}\right)$ and provides a quantitative estimate of focal lipid core size.

\section{Pre-PCI NIRS: assessing lesion length and minimizing geographic miss}

Incomplete lesion coverage at the time of stent 


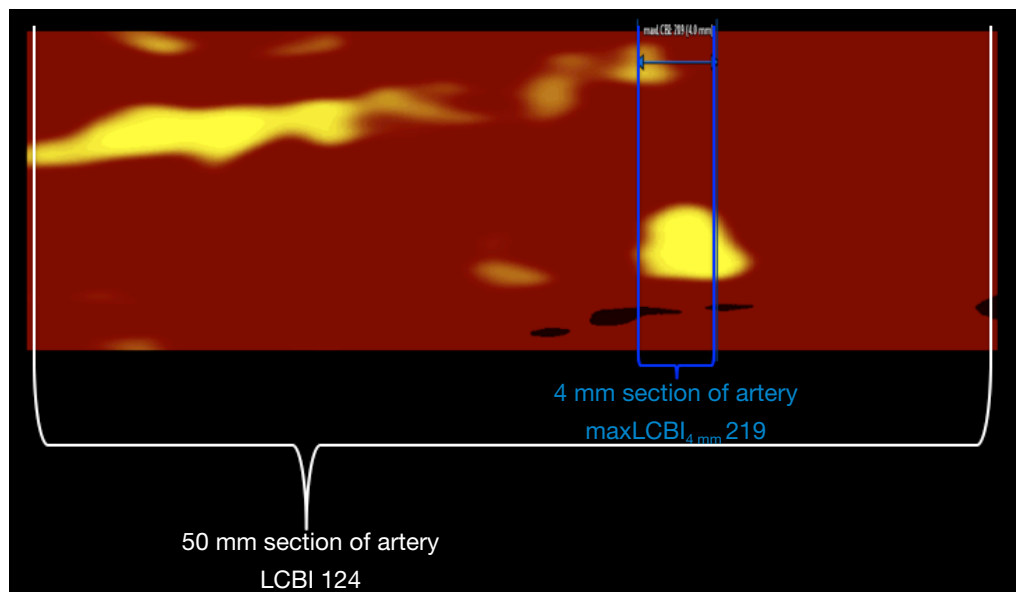

Figure 2 NIRS LCBI and maxLCBI ${ }_{4 m}$. Once NIRS imaging is performed, any region of interest on the chemogram can be selected by the operator and the software will automatically calculate the LCBI and maxLCBI $\mathrm{mm}_{\mathrm{m}}$ in the selected region. In the case above, the operator selected the $50-\mathrm{mm}$ segment of artery between the white lines as the region of interest. The LCBI, which is calculated as the number of yellow pixels divided by all valid pixels in the region of interest multiplied by 1,000, was 124 in this case. Upon selecting the region of interest (white lines), the software automatically identifies the maxLCBI ${ }_{4 \mathrm{~mm}}$, defined as the 4-mm segment within the region of interest having the greatest LCBI (blue lines). LCBI, lipid core burden index; maxLCBI ${ }_{4 m}$, maximum lipid core burden index in 4 mm; NIRS, nearinfrared spectroscopy.

implantation, known as longitudinal geographic miss, is associated with adverse outcomes after PCI (7). Pre-PCI NIRS imaging may complement IVUS or OCT imaging in reducing the likelihood of geographic miss, particularly in selecting the length of stent necessary to ensure adequate coverage of LRP at the margins of the target lesion. In prior NIRS studies, LRP has been shown to frequently extend beyond the angiographic margins of the target lesion $(8,9)$. In a study by Hanson et al., NIRS detected LRP extending beyond angiographic borders in 52\% of target lesions. The adverse consequences of leaving LRP uncovered at stent margins were recently suggested in a study by Ino et al., in which uncovered LRP at the stent margins, as detected by OCT, was associated with an increased risk of restenosis during follow up, with larger lipid cores being associated with greater risk (10). Additional studies are required to determine if lipid cores detected by NIRS in the stent margins are similarly associated with subsequent restenosis.

\section{Pre-PCI NIRS: predicting periprocedural myocardial infarction risk}

Intracoronary NIRS is capable of identifying lesions at an increased risk for causing periprocedural myocardial infarction during PCI. Utilizing data from the COLOR registry, Goldstein et al. reported target lesions with a maxLCBI $_{4 \mathrm{~mm}} \geq 500$ were associated with periprocedural myocardial infarction in $50 \%$ of cases (11). In contrast, the rate of periprocedural myocardial infarction among target lesions with a maxLCBI ${ }_{4 m}<500$ was only $4.2 \%$. The concept that target lesions having a large LRP detected by NIRS are associated with a greater risk of periprocedural myocardial infarction was confirmed in the CANARY trial (12). In the CANARY trial, the optimal threshold for lesions at risk of periprocedural myocardial infarction, as determined by receiver operating characteristic analysis, was a maxLCBI ${ }_{4 \mathrm{~mm}}$ value of 388 .

Ideally, being able to predict periprocedural myocardial infarction would enable preventative maneuvers to decrease risk. However, the CANARY trial, which randomized patients with target lesions having maxLCBI ${ }_{4 \mathrm{~mm}}$ values $\geq 600$ to undergo PCI with or without the use of a distal embolic protection device, showed that distal protection did not decrease the risk of periprocedural myocardial infarction (12).

\section{Post-PCI NIRS: stent optimization}

Considering the currently available NIRS device is a multimodality catheter providing simultaneous NIRS and IVUS imaging (Figure 3), and that the use of IVUS to 


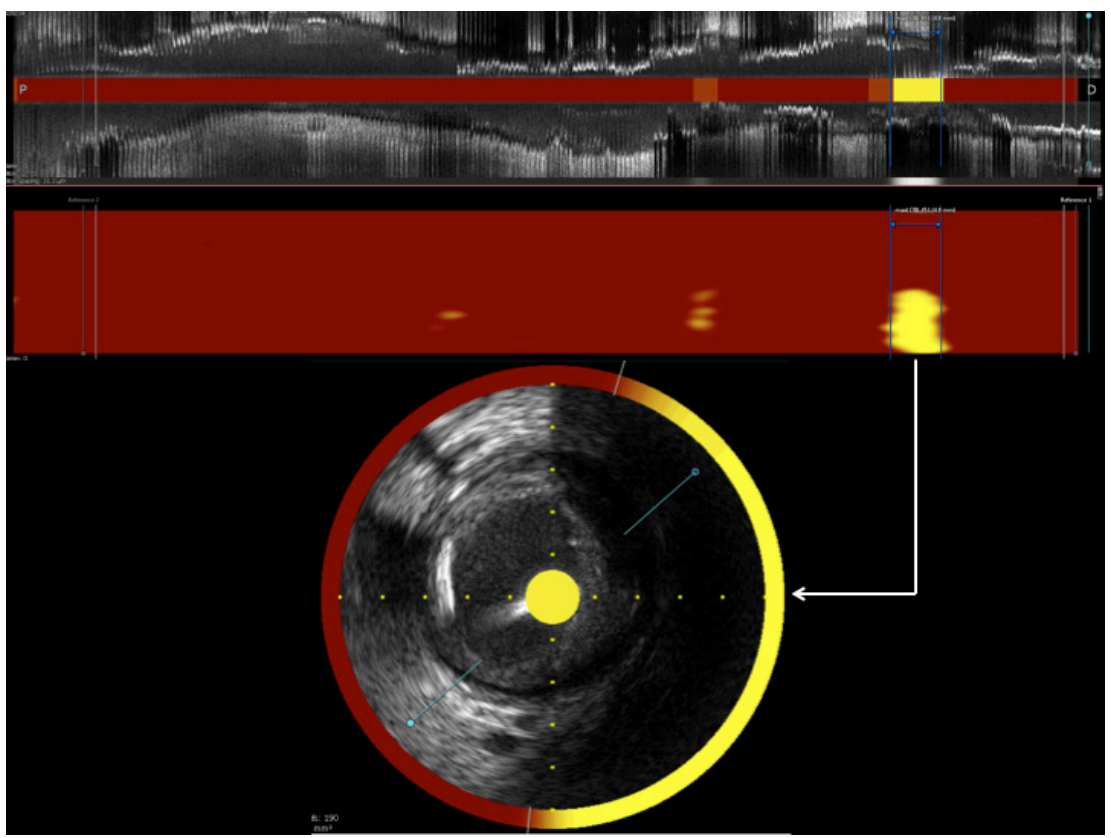

Figure 3 Multimodality imaging with the combined NIRS-IVUS catheter. Shown are combined NIRS-IVUS images obtained in a patient with coronary artery disease. The longitudinal IVUS image is shown at the top of the figure and reveals calcified plaque scattered throughout the vessel. The NIRS block chemogram is overlying the longitudinal IVUS image. The NIRS chemogram is shown beneath the longitudinal IVUS image and reveals a large LRP (yellow spot) in the distal vessel. Below the chemogram is a representative cross-sectional NIRS-IVUS image, the center of which corresponds to the block chemogram. The outer rim of the cross-sectional image corresponds to the chemogram, as shown by the white arrow. IVUS, intravascular ultrasound; LRP, lipid-rich plaque; NIRS, near-infrared spectroscopy.

guide PCI has been convincingly shown to improve clinical outcomes in multiple randomized controlled trials (13), the NIRS-IVUS device likely has a role in post-PCI imaging to optimize the stent result in a manner analogous to previous IVUS trials. Whereas post-PCI IVUS imaging can ensure adequate stent expansion and the absence of both dissection and large plaque burden at the stent edges, the role of postPCI NIRS in stent optimization remains to be defined. NIRS imaging of target lesions before and after stenting have shown a decrease in lipid burden after PCI $(14,15)$ (Figure 4). The mechanisms accounting for this decrease in lipid are likely multifactorial $(11,12,14,15)$, and in some cases are due to liberation and down-stream embolization of lipid debris (16). Apart from the potential risk of no-reflow and periprocedural myocardial infarction $(11,12)$, outcomes associated with the change in target lesion lipid burden or associated with the amount of retained lipid underlying the implanted stent have not yet been delineated. Interestingly, prior post-mortem observations have linked the implantation of stent struts into a lipid core with the occurrence of stent thrombosis $(17,18)$. Future studies may perhaps delineate if residual lipid in stented segments, as detected by NIRS after stent implantation, is a risk factor for subsequent target lesion failure.

\section{NIRS for the detection of vulnerable patients}

Several studies have now demonstrated NIRS imaging can be used to identify vulnerable patients who are at an increased risk of future patient-level cardiovascular events. In 2014, Oemrawsingh et al. were the first to show that NIRS findings in a non-target coronary artery were associated with an increased risk of major adverse cardiovascular events (MACE) (19). In this study, patients with an LCBI in a non-culprit vessel greater than the median value of 43 had a four-fold increased risk of MACE during 1 year of follow up. Among patients undergoing NIRS imaging in the target artery, it was subsequently demonstrated that a single non-culprit lesion having a maxLCBI ${ }_{4 \mathrm{~mm}} \geq 400$ was associated with an increased risk of major adverse cardiovascular and cerebrovascular events (20). This study also demonstrated a lipid burden- 


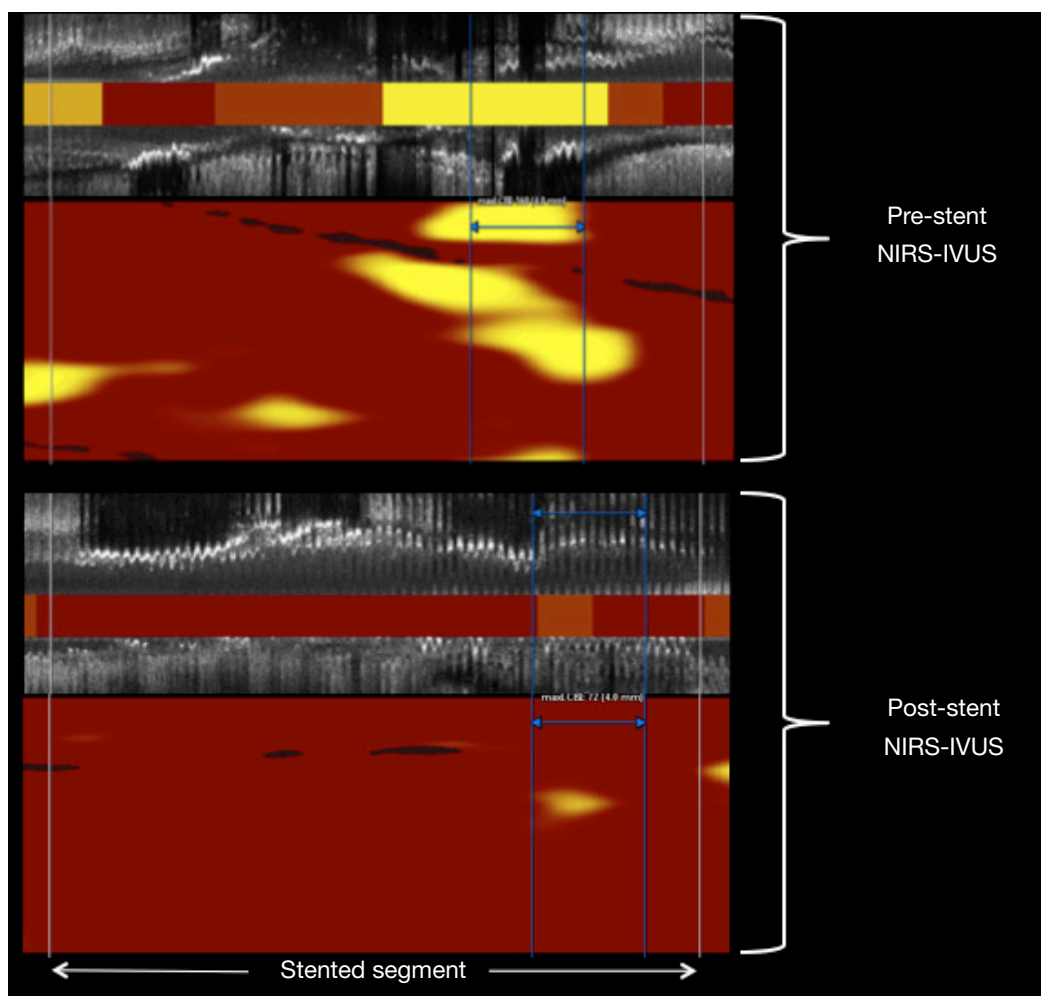

Figure 4 Reduction in NIRS lipid signal after stenting. Pre-stent NIRS-IVUS images (top) of the target lesion reveal a large LRP at the target site having a maxLCBI 4 mm of 560 (region between blue lines). Post-PCI NIRS-IVUS images (bottom) reveal a significant decrease in the target lesion lipid signal with a maxLCBI 4 mm of 72 (region between blue lines). IVUS, intravascular ultrasound; LRP, lipid-rich plaque; maxLCBI ${ }_{4 \mathrm{~mm}}$, maximum lipid core burden index in $4 \mathrm{~mm}$; NIRS, near-infrared spectroscopy; PCI, percutaneous coronary intervention.

dependent relationship wherein larger LRPs detected by NIRS, evidenced by increasing maxLCBI ${ }_{4 \mathrm{~mm}}$ values, were seemingly associated with greater risk. An independent and continuous relationship between increasing $\operatorname{maxLCBI}_{4 \mathrm{~mm}}$ values and increasing risk of MACE was demonstrated during 4 years of follow up in a study by Schuurman et al. (21).

The ability of NIRS to identify vulnerable patients was ultimately confirmed in the prospective, multicenter LRP study, in which $>1,200$ patients underwent multivessel NIRS-IVUS imaging at baseline and were followed for 2 years for the development of subsequent MACE (22). In this study, the continuous relationship between increasing maxLCBI ${ }_{4 \mathrm{~mm}}$ values and increasing MACE risk was confirmed. In non-culprit segments, for every 100-unit increase in the maxLCBI ${ }_{4 \mathrm{~mm}}$ value, there was an increased risk of MACE with an associated hazard ratio (HR) of $1.21(1.09-1.35, \mathrm{P}=0.0004)$. This study also confirmed the $\operatorname{maxLCBI}_{4 \mathrm{~mm}}$ threshold value of 400 as a predictor of patient-level risk; specifically, a maxLCBI ${ }_{4 m}>400$ in non-culprit segments was associated with a HR of 2.18 $(1.48-3.22, \mathrm{P}<0.0001)$ for patient-level MACE.

The ability of NIRS to identify patients at increased risk of MACE based on the detection of non-culprit lipid cores is in line with similar findings using other imaging modalities capable of detecting intracoronary lipid, including angioscopy and OCT $(23,24)$. Although the ability of NIRS technology to identify vulnerable patients has now been convincingly demonstrated, the translation of this knowledge into patient-specific preventative or interventional strategies has not yet been established. Future studies are needed to determine how best to guide therapy for patients deemed to be at high risk for patientlevel events based on NIRS imaging.

\section{NIRS for the detection of vulnerable plaques}

Post-mortem studies have identified fibroatheromas that are lipid-rich and thin-capped to be cardinal precursor lesions 


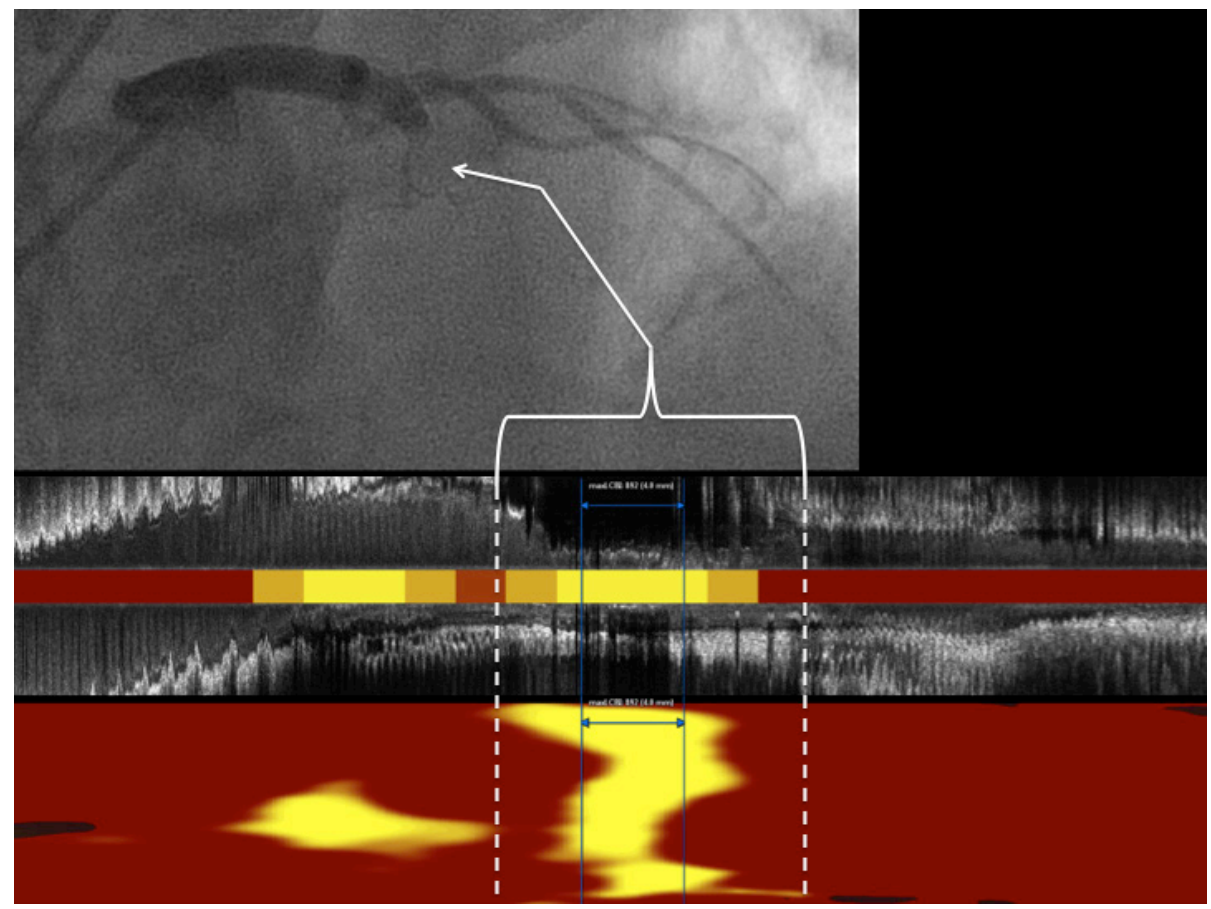

Figure 5 NIRS-IVUS imaging in a patient suffering an anterior STEMI. Invasive coronary angiography patient presenting with an acute anterior STEMI demonstrated complete occlusion of the proximal LAD (arrow). After reestablishing flow in the vessel with angioplasty, NIRS-IVUS imaging was performed. The culprit lesion is readily apparent on the longitudinal IVUS images (bracket and dashed white lines). NIRS revealed a large, nearly circumferential LRP at the culprit site having a maxLCBI ${ }_{4 m}$ of 892 . IVUS, intravascular ultrasound; LRP, lipid-rich plaque; maxLCBI ${ }_{4 m}$, maximum lipid core burden index in 4 mm; NIRS, near-infrared spectroscopy; STEMI, ST-segment elevation myocardial infarction; LAD, left anterior descending coronary artery.

of acute myocardial infarction $(25,26)$. An initial crosssectional study of patients undergoing NIRS imaging at the time of an ST-segment elevation myocardial infarction (STEMI) identified the majority of culprit lesions had a maxLCBI $_{4 \mathrm{~mm}}>400$ (27). This NIRS signature of culprit lesions has since been demonstrated across the spectrum of acute coronary events, including in culprit lesions responsible for STEMI, non-STEMI, unstable angina, and sudden cardiac death (27-32) (Figure 5). Considering the large lipid cores identified by NIRS in these culprit lesions were likely present well in advance of the coronary event, it has been hypothesized that the maxLCBI ${ }_{4 \mathrm{~mm}}$ metric might be able to identify non-culprit plaques at increased risk of site-specific future events.

In addition to evaluating the ability of NIRS to identify vulnerable patients, the LRP study also tested a vulnerable plaque hypothesis in which the association between nonculprit segment maxLCBI ${ }_{4 \mathrm{~mm}}$ at baseline and subsequent lesion-specific coronary events was investigated (22). The study was positive in this regard, as each 100-unit increase in maxLCBI $_{4 \mathrm{~mm}}$ at baseline was associated with a HR of $1.45(1.30-1.60, \mathrm{P}<0.0001)$ for a site-specific event during 2 years of follow-up. Furthermore, non-culprit lesions having a maxLCBI ${ }_{4 \mathrm{~mm}}>400$ at baseline were at a more than a four-fold risk of being associated with a coronary event over the next 24 months.

\section{Limitations of NIRS imaging}

A limitation of intracoronary NIRS imaging is that while its diffuse reflectance approach allows for notably less interference from proximal structures, such as stent struts, than other imaging modalities, the precise depth of the interrogated tissue is not known. This limitation was highlighted in a prior study using NIRS-IVUS imaging in an attempt to identify neoatherosclerosis (33). In this study, NIRS detected LRP within previously stented segments, but uncertainty existed whether the lipid cores 
identified were superficial to the stent, consistent with neoatherosclerosis, or deep to the stent, consistent with a lipid core in the wall of the vessel. The lack of structural imaging provided by NIRS represents another limitation and has been the impetus to combine NIRS imaging, which provides compositional information, with other imaging modalities that provide structural information.

\section{Future directions}

Based on the findings of the LRP study, the United States Food and Drug Administration (FDA) has now approved intracoronary NIRS imaging for the identification of both vulnerable patients and vulnerable plaques. Whereas the findings of the LRP study support the ability of NIRS imaging to identify vulnerable plaques, it has not yet been determined whether the predictive value associated with the NIRS findings is sufficiently high to warrant a change in local or systemic therapy for event prevention. These uncertainties are currently the focus of ongoing and future studies.

As stated above, NIRS does not provide structural imaging and therefore has not yet been used in clinical settings to assess the fibrous cap thickness overlying LRP. There are ongoing efforts to develop the ability of NIRS to evaluate fibrous cap thickness based on the collagen content of the fibrous cap and its respective spectral signal (34). Although NIRS is currently combined with IVUS, IVUS does not have sufficient special resolution to determine fibrous cap thickness. A combined OCT-NIRS catheter is in development (35) and since OCT has a spatial resolution capable of measuring fibrous cap thickness, the combined OCT-NIRS catheter will likely be capable of differentiating LRP detected by NIRS based on the fibrous cap thickness measured by OCT.

\section{Conclusions}

Intracoronary NIRS imaging has been developed and validated to detect LRP in the coronary arteries. The role of NIRS in optimizing PCI procedures continues to be studied. LRP detected by NIRS has been shown to be associated with both patient-level and site-specific future coronary events. NIRS is the first and only intracoronary imaging modality approved by the FDA for the detection of vulnerable patients and vulnerable plaques. The utility of intracoronary NIRS imaging in the clinical setting is likely to expand as ongoing and future research endeavors with
NIRS are completed.

\section{Acknowledgments}

Funding: None.

\section{Footnote}

Provenance and Peer Review: This article was commissioned by the Guest Editor (Dennis T. L. Wong) for the series "Intracoronary Imaging" published in Cardiovascular Diagnosis and Therapy. The article has undergone external peer review.

Conflicts of Interest: Both authors have completed the ICMJE uniform disclosure form (available at http://dx.doi. org/10.21037/cdt.2020.02.02). The series "Intracoronary Imaging" was commissioned by the editorial office without any funding or sponsorship. RDM reports grants and personal fees from Infraredx, personal fees from Spectrawave, during the conduct of the study. The other author has no other conflicts of interest to declare.

Ethical Statement: The authors are accountable for all aspects of the work in ensuring that questions related to the accuracy or integrity of any part of the work are appropriately investigated and resolved.

Open Access Statement: This is an Open Access article distributed in accordance with the Creative Commons Attribution-NonCommercial-NoDerivs 4.0 International License (CC BY-NC-ND 4.0), which permits the noncommercial replication and distribution of the article with the strict proviso that no changes or edits are made and the original work is properly cited (including links to both the formal publication through the relevant DOI and the license). See: https://creativecommons.org/licenses/by-nc-nd/4.0/.

\section{References}

1. Su JL, Grainger SJ, Greiner CA, et al. Detection and structural characterization of lipid-core plaques with intravascular NIRS-IVUS imaging. Interv Cardiol 2015;7:519-36.

2. Gardner CM, Tan H, Hull EL, et al. Detection of lipid core coronary plaques in autopsy specimens with a novel catheter-based near-infrared spectroscopy system. JACC Cardiovasc Imaging 2008;1:638-48. 
3. Yamada R, Okura H, Kume T, et al. Histological characteristics of plaque with ultrasonic attenuation: a comparison between intravascular ultrasound and histology. J Cardiol 2007;50:223-8.

4. Di Vito L, Imola F, Gatto L, et al. Limitations of OCT in identifying and quantifying lipid components: an in vivo comparison study with IVUS-NIRS. EuroIntervention 2017;13:303-11.

5. Negi SI, Didier R, Ota H, et al. Role of near-infrared spectroscopy in intravascular coronary imaging. Cardiovasc Revasc Med 2015;16:299-305.

6. Danek BA, Karatasakis A, Madder RD, et al. Experience with the multimodality near-infrared spectroscopy/ intravascular ultrasound coronary imaging system: principles, clinical experience, and ongoing studies. Curr Cardiovasc Imaging Rep 2016;9:7.

7. Kang SJ, Cho YR, Park GM, et al. Intravascular ultrasound predictors for edge restenosis after newer generation drug-eluting stent implantation. Am J Cardiol 2013;111:1408-14.

8. Dixon SR, Grines CL, Munir A, et al. Analysis of target lesion length before coronary artery stenting using angiography and near-infrared spectroscopy versus angiography alone. Am J Cardiol 2012;109:60-6.

9. Hanson ID, Goldstein JA, Dixon SR, et al. Comparison of coronary artery lesion length by NIRS-IVUS versus angiography alone. Coron Artery Dis 2015;26:484-9.

10. Ino Y, Kubo T, Matsuo Y, et al. Optical coherence tomography predictors for edge restenosis after everolimus-eluting stent implantation. Circ Cardiovasc Interv 2016. doi: 10.1161/ CIRCINTERVENTIONS.116.004231.

11. Goldstein JA, Maini B, Dixon SR, et al. Detection of lipidcore plaques by intracoronary near-infrared spectroscopy identifies high risk of periprocedural myocardial infarction. Circ Cardiovasc Interv 2011;4:429-37.

12. Stone GW, Maehara A, Muller JE, et al. Plaque characterization to inform the prediction and prevention of periprocedural myocardial infarction during percutaneous coronary intervention: the CANARY trial (coronary assessment by near-infrared of atherosclerotic ruptureprone yellow). JACC Cardiovasc Interv 2015;8:927-36.

13. Shin DH, Hong SJ, Mintz GS, et al. Effects of intravascular ultrasound-guided versus angiographyguided new-generation drug-eluting stent implantation: meta-analysis with individual patient-level data from 2,345 randomized patients. JACC Cardiovasc Interv 2016;9:2232-9.
14. Maini A, Buyantseva L, Maini B. In vivo lipid core plaque modification with percutaneous coronary revascularization: a near-infrared spectroscopy study. J Invasive Cardiol 2013;25:293-5.

15. Lu H, Tijssen RYG, Grundeken MJ, et al. Decrease in lipid rich plaque during percutaneous coronary intervention: reality or artefact? An observational study using near-infrared spectroscopy. Interv Cardiol 2017;9:281-7.

16. Brilakis ES, Abdel-Karim AR, Papayannis AC, et al. Embolic protection device utilization during stenting of native coronary artery lesions with large lipid core plaques as detected by near-infrared spectroscopy. Catheter Cardiovasc Interv 2012;80:1157-62.

17. Joner M, Finn AV, Farb A, et al. Pathology of drug-eluting stents in humans: delayed healing and late thrombotic risk. J Am Coll Cardiol 2006;48:193-202.

18. Nakano M, Yahagi K, Otsuka F, et al. Causes of early stent thrombosis in patients presenting with acute coronary syndrome: an ex vivo human autopsy study. J Am Coll Cardiol 2014;63:2510-20.

19. Oemrawsingh RM, Cheng JM, García-García HM, et al. Near-infrared spectroscopy predicts cardiovascular outcome in patients with coronary artery disease. $\mathrm{J}$ Am Coll Cardiol 2014;64:2510-8.

20. Madder RD, Husaini M, Davis AT, et al. Large lipid-rich coronary plaques detected by near-infrared spectroscopy at non-stented sites in the target artery identify patients likely to experience future major adverse cardiovascular events. Eur Heart J Cardiovasc Imaging 2016;17:393-9.

21. Schuurman AS, Vroegindewey M, Kardys I, et al. Nearinfrared spectroscopy-derived lipid core burden index predicts adverse cardiovascular outcome in patients with coronary artery disease during long-term follow-up. Eur Heart J 2018;39:295-302.

22. Waksman R, Di Mario C, Torguson R, et al. Identification of patients and plaques vulnerable to future coronary events with near-infrared spectroscopy intravascular ultrasound imaging: a prospective, cohort study. Lancet 2019;394:1629-37.

23. Ohtani T, Ueda Y, Mizote I, et al. Number of yellow plaques detected in a coronary artery is associated with future risk of acute coronary syndrome: detection of vulnerable patients by angioscopy. J Am Coll Cardiol 2006;47:2194-200.

24. Prati F, Romagnoli E, Gatto L, et al. Relationship between coronary plaque morphology of the left anterior descending artery and 12 months clinical outcome: the 
CLIMA study. Eur Heart J 2020;41:383-91.

25. Burke AP, Farb A, Malcom GT, et al. Coronary risk factors and plaque morphology in men with coronary disease who died suddenly. N Engl J Med 1997;336:1276-82.

26. Farb A, Tang AL, Burke AP, et al. Sudden coronary death. Frequency of active coronary lesions, inactive coronary lesions, and myocardial infarction. Circulation 1995;92:1701-9.

27. Madder RD, Goldstein JA, Madden SP, et al. Detection by near-infrared spectroscopy of large lipid core plaques at culprit sites in patients with acute ST-segment elevation myocardial infarction. JACC Cardiovasc Interv 2013;6:838-46.

28. Madder RD, Puri R, Muller JE, et al. Confirmation of the intracoronary near-infrared spectroscopy threshold of lipid-rich plaques that underlie ST-segment-elevation myocardial infarction. Arterioscler Thromb Vasc Biol 2016;36:1010-5.

29. Madder RD, Husaini M, Davis AT, et al. Detection by near-infrared spectroscopy of large lipid cores at culprit sites in patients with non-ST-segment elevation myocardial infarction and unstable angina. Catheter Cardiovasc Interv 2015;86:1014-21.

30. Madder RD, Wohns DH, Muller JE. Detection by

Cite this article as: Wilkinson SE, Madder RD. Intracoronary near-infrared spectroscopy-role and clinical applications. Cardiovasc Diagn Ther 2020;10(5):1508-1516. doi: 10.21037/ cdt.2020.02.02 intracoronary near-infrared spectroscopy of lipid core plaque at culprit sites in survivors of cardiac arrest. J Invasive Cardiol 2014;26:78-9.

31. Madder RD, Smith JL, Dixon SR, et al. Composition of target lesions by near-infrared spectroscopy in patients with acute coronary syndrome versus stable angina. Circ Cardiovasc Interv 2012;5:55-61.

32. Karlsson S, Anesäter E, Fransson K, et al. Intracoronary near-infrared spectroscopy and the risk of future cardiovascular events. Open Heart 2019;6:e000917.

33. Madder RD, Khan M, Husaini M, et al. Combined near-infrared spectroscopy and intravascular ultrasound imaging of pre-existing coronary artery stents: can nearinfrared spectroscopy reliably detect neoatherosclerosis? Circ Cardiovasc Imaging 2016. doi: 10.1161/ CIRCIMAGING.115.003576.

34. Madden S, Raichlen J, Muller J. Spectroscopic detection of fibrous cap thickness overlying lipid core coronary plaques with a catheter-based near-infrared spectroscopy system. J Am Coll Cardiol 2012;59:E308.

35. Fard AM, Vacas-Jacques P, Hamidi E, et al. Optical coherence tomography--near infrared spectroscopy system and catheter for intravascular imaging. Opt Express 2013;21:30849-58. 